CERN-PPE/97-54

12 May 1997

\title{
CPLEAR results on the CP parameters of neutral kaons decaying to $\pi^{+} \pi^{-} \pi^{0}$
}

\author{
The CPLEAR Collaboration
}

R. Adler ${ }^{2}$, A. Angelopoulos ${ }^{1}$, A. Apostolakis ${ }^{1}$, E. Aslanides ${ }^{11}$, G. Backenstoss ${ }^{2}$, P. Bargassa ${ }^{13}$, C.P. Bee ${ }^{9}$, O. Behnke ${ }^{17}$, A. Benelli ${ }^{9}$, V. Bertin ${ }^{11}$, F. Blanc ${ }^{7,13}$, P. Bloch ${ }^{4}$, P. Carlson ${ }^{15}$, M. Carroll ${ }^{9}$, J. Carvalho ${ }^{5}$, E. Cawley ${ }^{9}$, S. Charalambous ${ }^{16}$, G. Chardin ${ }^{14}$, M.B. Chertok ${ }^{3}$, A. Cody ${ }^{9}$, M. Danielsson ${ }^{15}$, M. Dejardin ${ }^{14}$, J. Derre ${ }^{14}$, A. Ealet $^{11}$, B. Eckart ${ }^{2}$, C. Eleftheriadis ${ }^{16}$, I. Evangelou ${ }^{8}$, L. Faravel ${ }^{7}$, P. Fassnacht ${ }^{11}$, C. Felder ${ }^{2}$, R. Ferreira-Marques ${ }^{5}$, W. Fetscher ${ }^{17}$, M. Fidecaro ${ }^{4}$, A. Filipčič ${ }^{10}$, D. Francis ${ }^{3}$, J. Fry ${ }^{9}$, E. Gabathuler ${ }^{9}$, R. Gamet ${ }^{9}$, D. Garreta ${ }^{14}$, H.- J. Gerber ${ }^{17}$, A. Go ${ }^{14}$, C. Guyot $^{14}$, A. Haselden ${ }^{9}$, P.J. Hayman ${ }^{9}$, F. Henry-Couannier ${ }^{11}$, R.W. Hollander ${ }^{6}$, E. Hubert ${ }^{11}$, K. Jon-And ${ }^{15}$, P.-R. Kettle ${ }^{13}$, C. Kochowski ${ }^{14}$, P. Kokkas ${ }^{4}$, R. Kreuger ${ }^{6,13}$, R. Le Gac ${ }^{11}$, F. Leimgruber ${ }^{2}$, A. Liolios ${ }^{16}$, E. Machado ${ }^{5}$, I. Mandić10 ${ }^{10}$ N. $\operatorname{Manthos}^{8}$, G. $\operatorname{Marel}^{14}$, M. Mikuž ${ }^{10}$, J. Miller ${ }^{3}$, F. Montanet ${ }^{11}$, A. Muller ${ }^{14}$, T. Nakada ${ }^{13}$, B. Pagels ${ }^{17}$, I. Papadopoulos ${ }^{16}$, P. Pavlopoulos ${ }^{2}$, J. Pinto da Cunha ${ }^{5}$, A. Policarpo ${ }^{5}$, G. Polivka ${ }^{2}$, R. Rickenbach ${ }^{2}$, B.L. Roberts ${ }^{3}$, T. Ruf $^{4}$, L. Sakeliou ${ }^{1}$, P. Sanders ${ }^{9}$, C. Santoni ${ }^{2}$, M. Schäfer ${ }^{17}$, L.A. Schaller ${ }^{7}$, T. Schietinger ${ }^{2}$, A. Schopper ${ }^{4}$, P. Schune ${ }^{14}$, A. Soares ${ }^{14}$, L. Tauscher ${ }^{2}$, C. Thibault $^{12}$, F. Touchard ${ }^{11}$, C. Touramanis ${ }^{4}$, F. Triantis ${ }^{8}$, E. Van Beveren ${ }^{5}$, C.W.E. Van Eijk ${ }^{6}$, S. Vlachos ${ }^{2}$, P. Weber ${ }^{17}$, O. Wigger ${ }^{13}$, M. Wolter ${ }^{17}$, C. Yeche ${ }^{14}$, D. Zavrtanik ${ }^{10}$ and D. Zimmerman ${ }^{3}$

\begin{abstract}
The CPLEAR experiment measured time-dependent decay-rate asymmetries of $\mathrm{K}^{0}$ and $\overline{\mathrm{K}}^{0}$ decaying to $\pi^{+} \pi^{-} \pi^{0}$ in order to study the interference between the decay amplitudes of $\mathrm{K}_{\mathrm{S}}^{0}$ - either CP-violating or CP-conserving - and the $\mathrm{CP}$-conserving $\mathrm{K}_{\mathrm{L}}^{0}$ decay amplitude. From the analysis of the complete data set we find for the CP-violating parameter $\eta_{+-0}$, $\operatorname{Re}\left(\eta_{+-0}\right)=\left(-2 \pm 7\right.$ stat. ${ }_{-1}^{+4}$ syst. $) \times 10^{-3}, \operatorname{Im}\left(\eta_{+-0}\right)=\left(-2 \pm 9\right.$ stat. ${ }_{-1}^{+2}$ syst. $) \times$ $10^{-3}$ and for the CP-conserving parameter $\lambda, \operatorname{Re}(\lambda)=(+28 \pm 7$ stat. \pm 3 syst. $) \times 10^{-3}$, $\operatorname{Im}(\lambda)=(-10 \pm 8$ stat. \pm 2 syst. $) \times 10^{-3}$. From the latter, the branching ratio of the CPconserving $\mathrm{K}_{\mathrm{S}}^{0} \rightarrow \pi^{+} \pi^{-} \pi^{0}$ decay is deduced to be $\mathrm{B}=\left(2.5_{-1.0}^{+1.3}\right.$ stat. ${ }_{-0.6}^{+0.5}$ syst. $) \times 10^{-7}$.
\end{abstract}


The CPLEAR experiment at the Low Energy Antiproton Ring at CERN uses tagged $\mathrm{K}^{0}$ and $\overline{\mathrm{K}}^{0}$ to study CP, T and CPT symmetries in the neutral kaon system. In previous CPLEAR publications, the measurements of the $\mathrm{CP}$-violating parameter $\eta_{+-0}[1]$ and the value of the $\mathrm{CP}$ conserving parameter $\lambda$ [2], obtained from the analysis of neutral kaons decaying into $\pi^{+} \pi^{-} \pi^{0}$, were reported for a $25 \%$ data sample. In this letter, new measurements of $\eta_{+-0}$ and $\lambda$ based on our complete data set are presented.

Interest in the search of the CP-violating amplitude of $\mathrm{K}_{\mathrm{S}}^{0} \rightarrow \pi^{+} \pi^{-} \pi^{0}$ extends beyond the search for $\mathrm{CP}$ violation in the $\mathrm{K}_{\mathrm{S}}^{0}$ decays. Firstly, increasing experimental precision in the measurement of the phase, $\varphi_{+-}$, of the CP-violating parameter $\eta_{+-}$can be fully exploited for an indirect CPT test, only if the precision of the CP-violating parameters of neutral kaons decaying to three pions is improved substantially [3]. Secondly, the measurement of the CP-conserving $\mathrm{K}_{\mathrm{S}}^{0} \rightarrow \pi^{+} \pi^{-} \pi^{0}$ amplitude allows the results to be cross-checked with the phenomenological global fits to all known neutral- and charged-kaon decay rates and to be compared with the predictions of chiral perturbation theories [4-8].

The CP eigenvalue for the $\pi^{+} \pi^{-} \pi^{0}$ final state is given by $(-1)^{l+1}$ where $l$ is the relative angular momentum between the two charged pions (or between the $\pi^{0}$ and the $\pi^{+} \pi^{-}$pair). As the sum of the masses of the three pions is close to the kaon mass, the pions have a low kinetic energy $E_{\mathrm{CM}}(\pi)$ in the kaon rest-frame, and the states with $l>0$ are suppressed by the centrifugal barrier. This implies two contributions to the $\mathrm{K}_{\mathrm{S}}^{0} \rightarrow \pi^{+} \pi^{-} \pi^{0}$ decay amplitude $A_{\mathrm{S}}^{+-0}$ : one from the decay to a $\pi^{+} \pi^{-} \pi^{0}$ state with $\mathrm{CP}=+1$ (kinematics-suppressed and CP-allowed), and the other from the decay to a $\mathrm{CP}=-1$ state (kinematics-favoured but CP-suppressed). The $\mathrm{K}_{\mathrm{L}}^{0} \rightarrow \pi^{+} \pi^{-} \pi^{0}$ decay is dominated by the CP-allowed decay amplitude $A_{\mathrm{L}}^{+-0}$ with $l=0$ and $\mathrm{CP}=-1$.

The $\mathrm{K}_{\mathrm{L}}^{0}$ decay amplitude $A_{\mathrm{L}}^{+-0}$ interferes with both the $\mathrm{CP}$-conserving $\mathrm{K}_{\mathrm{S}}^{0}$ decay amplitude $A_{\mathrm{S}}^{3 \pi(\mathrm{CP}=+1)}$ and the $\mathrm{CP}$-violating $\mathrm{K}_{\mathrm{S}}^{0}$ decay amplitude $A_{\mathrm{S}}^{3 \pi(\mathrm{CP}=-1)}$. These amplitudes depend on the Dalitz variables $X$ and $Y$ [2], which can be estimated to be $X \simeq\left(2 m_{\mathrm{K}} / m_{\pi}^{2}\right)\left(E_{\mathrm{CM}}\left(\pi^{+}\right)-\right.$ $\left.E_{\mathrm{CM}}\left(\pi^{-}\right)\right)$and $Y \simeq\left(2 m_{\mathrm{K}} / m_{\pi}^{2}\right)\left(m_{\mathrm{K}} / 3-E_{\mathrm{CM}}\left(\pi^{0}\right)\right)$. The former interference term is antisymmetric in $X$, and is observed by separating the events with $X>0$ and $X<0$. We define the $\mathrm{CP}$-conserving parameter $\lambda$ as

$\lambda=\frac{\int_{X>0} d X d Y A_{\mathrm{L}}^{*}(X, Y) A_{\mathrm{S}}^{3 \pi(\mathrm{CP}=+1)}(X, Y)}{\int_{X>0} d X d Y\left|A_{\mathrm{L}}(X, Y)\right|^{2}}=-\frac{\int_{X<0} d X d Y A_{\mathrm{L}}^{*}(X, Y) A_{\mathrm{S}}^{3 \pi(\mathrm{CP}=+1)}(X, Y)}{\int_{X<0} d X d Y\left|A_{\mathrm{L}}(X, Y)\right|^{2}}$.

\footnotetext{
1) University of Athens, Greece

2) University of Basle, Switzerland

3) Boston University, USA

4) CERN, Geneva, Switzerland

5) LIP and University of Coimbra, Portugal

6) Delft University of Technology, Netherlands

7) University of Fribourg, Switzerland

8) University of Ioannina, Greece

9) University of Liverpool, UK

10) J. Stefan Inst. and Phys. Dep., University of Ljubljana, Slovenia

11) CPPM, IN2P3-CNRS et Université d'Aix-Marseille II, Marseille, France

12) CSNSM, IN2P3-CNRS, Orsay, France

13) Paul-Scherrer-Institut(PSI), Villigen, Switzerland

14) CEA, DSM/DAPNIA CE-Saclay, France

15) KTH-Stockholm, Sweden

16) University of Thessaloniki, Greece

17) ETH-IPP Zürich, Switzerland
} 
This contribution vanishes when data are integrated over the whole phase space, allowing the observation of the latter interference term through the measurement of the CP-violating parameter $\eta_{+-0}$ given by

$$
\eta_{+-0}=\frac{\int d X d Y A_{\mathrm{L}}^{*}(X, Y) A_{\mathrm{S}}^{3 \pi(\mathrm{CP}=-1)}(X, Y)}{\int d X d Y\left|A_{\mathrm{L}}(X, Y)\right|^{2}} .
$$

We define the decay rates for an initial $\mathrm{K}^{0}$ decaying to $\pi^{+} \pi^{-} \pi^{0}$ to be $R_{+}(\tau)$ and $R_{-}(\tau)$ for $X>0$ and $X<0$ respectively, where $\tau$ is the decay eigentime. Similarly the decay rates for an initial $\overline{\mathrm{K}}^{0}$ are defined as $\bar{R}_{+}(\tau)$ and $\bar{R}_{-}(\tau)$. The time-dependent asymmetry integrated over the whole phase space,

$$
\begin{aligned}
A_{+-0}(\tau) & =\frac{\left[\bar{R}_{+}(\tau)+\bar{R}_{-}(\tau)\right]-\left[R_{+}(\tau)+R_{-}(\tau)\right]}{\left[\bar{R}_{+}(\tau)+\bar{R}_{-}(\tau)\right]+\left[R_{+}(\tau)+R_{-}(\tau)\right]} \\
& =2 \operatorname{Re}(\varepsilon)-2 e^{-\Delta \Gamma \tau / 2}\left[\operatorname{Re}\left(\eta_{+-0}\right) \cos (\Delta m \tau)-\operatorname{Im}\left(\eta_{+-0}\right) \sin (\Delta m \tau)\right]
\end{aligned}
$$

where $\Delta m$ is the $\mathrm{K}_{\mathrm{L}}^{0}-\mathrm{K}_{\mathrm{S}}^{0}$ mass difference, $\Delta \Gamma$ the $\mathrm{K}_{\mathrm{S}}^{0}-\mathrm{K}_{\mathrm{L}}^{0}$ decay-width difference and $\varepsilon$ the CPviolation parameter in the $\mathrm{K}^{0}-\overline{\mathrm{K}}^{0}$ mixing, is used to extract the $\mathrm{CP}$-violation parameter $\eta_{+-0}$.

The two asymmetries obtained by separating the rates for $X>0$ and $X<0$,

$$
\begin{aligned}
A_{ \pm}(\tau) & =\frac{\bar{R}_{ \pm}(\tau)-R_{ \pm}(\tau)}{\bar{R}_{ \pm}(\tau)+R_{ \pm}(\tau)} \\
& =2 \operatorname{Re}(\varepsilon)-2 e^{-\Delta \Gamma \tau / 2}\left[\operatorname{Re}\left(\eta_{+-0} \pm \lambda\right) \cos (\Delta m \tau)-\operatorname{Im}\left(\eta_{+-0} \pm \lambda\right) \sin (\Delta m \tau)\right]
\end{aligned}
$$

are used to determine the $\mathrm{CP}$-conserving parameter $\lambda$.

\section{Data selection}

The CPLEAR method and detector have been described elsewhere [9]. In this experiment, initial $\mathrm{K}^{0}$ and $\overline{\mathrm{K}}^{0}$ are produced in the reactions

$$
\mathrm{p} \overline{\mathrm{p}}(\text { at rest }) \rightarrow \mathrm{K}^{+} \pi^{-} \overline{\mathrm{K}}^{0} \text { and } \mathrm{p} \overline{\mathrm{p}}(\text { at rest }) \rightarrow \mathrm{K}^{-} \pi^{+} \mathrm{K}^{0},
$$

where the strangeness of the neutral kaon is identified on an event-by-event basis.

The results reported in the present paper are based on the complete data set recorded by CPLEAR. The selection criteria are mostly identical to those described in our previous papers $[1,2]$. The differences are due to upgrades of our detector in 1994 and 1995. During the 1994 data-taking, the spherical target (radius $7 \mathrm{~cm}$ ) was replaced by a cylindrical one with a radius of $1.1 \mathrm{~cm}$, filled with gaseous hydrogen at a pressure of 27 bar. In 1995, a cylindrical proportional chamber with a radius of $1.5 \mathrm{~cm}$ was added around the new target. This chamber, located close to the annihilation vertex, was used in the first stage of the trigger to reject more efficiently the annihilation background, resulting in a smaller dead time and in an increase in the rate of recorded signal events by a factor of two.

In addition, an improvement of the selection criteria was possible by requiring in the new chamber a hit on each of the $\mathrm{K}^{ \pm} \pi^{\mp}$ tracks coming from the $\mathrm{p} \overline{\mathrm{p}}$ annihilation vertex, and no hit associated with the secondary particles from the neutral-kaon decay. This new condition allowed the release of some selection cuts used in the previous analysis in order to reject $\mathrm{p} \overline{\mathrm{p}} \rightarrow$ $\mathrm{K}^{+} \mathrm{K}^{-} \pi^{+} \pi^{-}$and $\mathrm{p} \overline{\mathrm{p}} \rightarrow \pi^{0} \mathrm{~K}^{+} \pi^{-} \overline{\mathrm{K}}^{0}$ (or c.c.) background. This leads to a substantial increase (about 30\%) in the signal acceptance at short decay time.

However, the increased material around the interaction region enhanced the background due to $\overline{\mathrm{K}}^{0} \mathrm{n} \rightarrow$ hyperon production followed by $\Lambda(1115) \rightarrow \mathrm{p} \pi^{-}$decay. Such events only appear 
in the $\overline{\mathrm{K}}^{0}$ data set, simulating CP-violation asymmetry. This background is strongly reduced by using time-of-flight to identify the protons.

The final data sample contains a total of 508000 events with a decay time $\tau>0.25 \tau_{\mathrm{S}}$, where $\tau_{\mathrm{S}}$ is the $\mathrm{K}_{\mathrm{S}}^{0}$ mean life. The decay-time distributions of initial $\mathrm{K}^{0}$ and $\overline{\mathrm{K}}^{0}$ are denoted by $N(\tau)$ and $\bar{N}(\tau)$ respectively. Figure 1 shows the measured decay-time distribution $N(\tau)+\bar{N}(\tau)$ of the full data set, together with the simulated decay-time distribution which includes $\mathrm{K}_{\mathrm{L}}^{0} \rightarrow$ $\pi^{+} \pi^{-} \pi^{0}$ events and the contribution from semileptonic decays. The simulated distribution is normalized to the real data for decay-times above $6 \tau_{\mathrm{S}}$, where semileptonic decays are the only source of background, by fitting a constant to the ratio of these two decay-time distributions.

The total-background fraction distribution $\zeta(\tau)$, defined in [1] as the relative difference between the data and the simulated $\pi^{+} \pi^{-} \pi^{0}$ events, is parametrized by

$$
\zeta(\tau)=\exp (-(0.2+4.1 \times \tau))-0.008+0.006 \times \tau .
$$

The linear part describes the semileptonic contribution which amounts to $4.15 \%$ of the selected events. The exponential part accounts for the background at short decay time $(0.16 \%$, possibly resulting from $\mathrm{K}^{+} \mathrm{K}^{-} \pi^{+} \pi^{-}$and $\pi^{0} \mathrm{~K}^{+} \pi^{-} \overline{\mathrm{K}}^{0}$ (or c.c.) events, $\mathrm{K}_{\mathrm{S}}^{0}$ decays into $\pi^{0} \pi^{0}$ followed by a $\pi^{0}$ Dalitz decay, and $\Lambda(1115) \rightarrow \mathrm{p} \pi^{-}$decays $)$.

\section{$3 \quad$ Decay asymmetries and fitting}

We followed the procedure described in Ref. [1], taking into account normalization (i.e. the tagging efficiency of $\overline{\mathrm{K}}^{0}$ relative to $\mathrm{K}^{0}$ ), regeneration and acceptance effects, to compute the measured asymmetry

$$
\begin{aligned}
\mathrm{A}_{+-0}^{\exp }(\tau) & =\frac{\bar{N}(\tau)-N(\tau)}{\bar{N}(\tau)+N(\tau)} \\
& \cong\left(\frac{\xi-1}{\xi+1}\right)+\frac{4 \xi[1-\zeta(\tau)]}{(\xi+1)^{2}} \mathrm{~A}_{+-0}(\tau)
\end{aligned}
$$

where $\mathrm{A}_{+-0}(\tau)$ is given by Eq. (2) and $\xi$ is the average $\overline{\mathrm{K}}^{0} / \mathrm{K}^{0}$ normalization factor. The parameter $\eta_{+-0}$ and the normalization factor $\xi$ are left free when fitting Eq. (4) to the data. The current world average values are used for $\Delta m$ and $\Gamma_{\mathrm{S}}[10]$, and $\Gamma_{\mathrm{L}}$ and $\operatorname{Re}(\varepsilon)$ [11].

The fit yields

$$
\begin{aligned}
& \operatorname{Re}\left(\eta_{+-0}\right)=(-2 \pm 7 \text { stat. }) \times 10^{-3} \\
& \operatorname{Im}\left(\eta_{+-0}\right)=(-2 \pm 9 \text { stat. }) \times 10^{-3} \\
& \xi \quad=1.116 \pm 0.004
\end{aligned}
$$

with a statistical correlation coefficient of the parameters $\operatorname{Re}\left(\eta_{+-0}\right)$ and $\operatorname{Im}\left(\eta_{+-0}\right)$ of $68 \%$. Figure 2 shows the measured asymmetry and the results of the fit.

In an analogous way, we followed the procedure of Ref. [2] to compute the measured decay-rate asymmetries for $X>0$ and $X<0$ :

$$
\begin{aligned}
A_{ \pm}^{\exp }(\tau) & =\frac{\bar{N}_{ \pm}(\tau)-N_{ \pm}(\tau)}{\bar{N}_{ \pm}(\tau)+N_{ \pm}(\tau)} \\
& \cong\left(\frac{\xi-1}{\xi+1}\right)+\frac{4 \xi[1-\zeta(\tau)]}{(\xi+1)^{2}} A_{ \pm}(\tau)
\end{aligned}
$$

where $A_{ \pm}(\tau)$ are the asymmetries given by Eq. (3). We first performed a six-parameter fit for the variables $\eta_{+-0}, \lambda$, and the normalization factors for events with positive and negative $X$, 
$\xi_{X>0}$ and $\xi_{X<0}$ respectively. This fit gives for $\eta_{+-0}$ values consistent with those of Eq. (5) and not correlated with $\lambda$. It also gives values of $\xi_{X>0}$ and $\xi_{X<0}$ which are statistically compatible. We therefore assumed $\xi_{X>0}=\xi_{X<0}=\xi$, and, in order to improve the determination of the normalization factor $\xi$, we fixed $\eta_{+-0}=\eta_{+-}$[11]. Simultaneous fits of the two asymmetries $A_{ \pm}^{\exp }(\tau)$ were then performed allowing only $\lambda$ and $\xi$ to vary. The fit gives

$$
\begin{aligned}
& \operatorname{Re}(\lambda)=(+28 \pm 7 \text { stat. }) \times 10^{-3} \\
& \operatorname{Im}(\lambda)=(-10 \pm 8 \text { stat. }) \times 10^{-3} \\
& \xi \quad=1.116 \pm 0.003
\end{aligned}
$$

with a statistical correlation coefficient of the parameters $\operatorname{Re}(\lambda)$ and $\operatorname{Im}(\lambda)$ of $68 \%$ and a negligible correlation between $\lambda$ and $\xi$. Figure 3 shows the measured asymmetries and the result of the fit.

\section{Systematic errors}

Tables 1 and 2 summarize the sources of systematic errors which may affect the determination of $\eta_{+-0}$ and $\lambda$.

Table 1: Summary of systematic errors on the real and imaginary parts of $\eta_{+-0}$

\begin{tabular}{|l|c|c|}
\hline Source of systematic error & $\operatorname{Re}\left(\eta_{+-0}\right) \times 10^{+3}$ & $\operatorname{Im}\left(\eta_{+-0}\right) \times 10^{+3}$ \\
\hline \multirow{2}{*}{ Amount and normalization of the background } & +1.5 & +0.7 \\
& -0.7 & -0.2 \\
\hline$\Lambda(1115)$ background & +4.1 & +1.9 \\
\hline CP-conserving K $\mathrm{S}_{\mathrm{S}}^{0}$ decay amplitude & -0.1 & - \\
\hline Decay-time dependence of normalization ${ }^{*}$ & \pm 0.3 & \pm 0.4 \\
Decay-time resolution & \pm 0.2 & \pm 0.2 \\
\hline Regeneration & $<0.1$ & $<0.1$ \\
\hline$\Delta m, \Gamma_{\mathrm{S}}$ and $\Gamma_{\mathrm{L}}$ & - & - \\
\hline
\end{tabular}

* Error determination limited by Monte Carlo statistics.

Table 2: Summary of systematic errors on the real and imaginary parts of $\lambda$

\begin{tabular}{|l|c|c|}
\hline Source of systematic error & $\operatorname{Re}(\lambda) \times 10^{+3}$ & $\operatorname{Im}(\lambda) \times 10^{+3}$ \\
\hline \multirow{2}{*}{ Amount of background and background asymmetry in $X$} & +1.3 & +0.5 \\
& -1.4 & -0.3 \\
\hline \multirow{2}{*}{$(1115)$ background and asymmetry in $X$} & +0.7 & +0.2 \\
& -2.3 & -0.8 \\
\hline Decay-time dependence of normalization* & \pm 0.2 & \pm 0.2 \\
Decay-time resolution & +1.1 & +0.7 \\
\hline Regeneration & $<0.1$ & $<0.1$ \\
\hline \multirow{2}{*}{$\Delta m, \Gamma_{\mathrm{S}}$ and $\Gamma_{\mathrm{L}}$} & \pm 0.1 & +0.1 \\
\hline Acceptance & \pm 2.0 & -0.2 \\
\hline
\end{tabular}

* Error determination limited by Monte Carlo statistics. 
- Amount of background

The amount and shape of the background at short decay time are estimated by comparing the decay-time distributions of real data and of simulated events. This determination is limited by the accuracy of our simulation. By comparing evaluations performed either on the decay-time or on the decay-radius distributions, and by varying the region where the simulated data are normalized to the real data, the total-background fraction at short decay time is estimated with an error of $+2 \times 10^{-3}$.

- Normalization of background

The $\overline{\mathrm{K}}^{0} / \mathrm{K}^{0}$ normalization factor for background events at short decay time may be different to signal events. This contribution to the systematic error was estimated by using the normalization either of the rejected $\mathrm{K}^{+} \mathrm{K}^{-} \pi^{+} \pi^{-}$events or of the rejected $\pi^{0} \mathrm{~K}^{+} \pi^{-} \overline{\mathrm{K}}^{0}$ (or c.c.) events for the maximum amount of background computed at short decay time.

\section{- Background asymmetry in X}

The $\overline{\mathrm{K}}^{0} / \mathrm{K}^{0}$ normalization factor for background events may depend on the variable $X$. This is the case for the $\pi^{0} \mathrm{~K}^{+} \pi^{-} \overline{\mathrm{K}}^{0}$ (or c.c.) background where the inversion of the primary pion with the same-sign secondary pion introduces a systematic shift of $X$ which is positive for a $\mathrm{K}^{0}$-tagged event and negative for a $\overline{\mathrm{K}}^{0}$ event. From the rejected $\pi^{0} \mathrm{~K}^{+} \pi^{-} \overline{\mathrm{K}}^{0}$ (or c.c.) events we estimated that the difference between the normalization factors for background events at $X>0$ and $X<0$ was less than $20 \%$. The resulting systematic error was determined by applying this normalization asymmetry to the estimated maximum amount of background at short decay time.

- The $\Lambda$ (1115) background

The remaining $\Lambda(1115) \rightarrow \mathrm{p} \pi^{-}$component, which only contributes to the $\overline{\mathrm{K}}^{0}$ data set, may be asymmetric in $X$ because of the misidentification of the proton as a pion. These events were estimated to be less than $0.019 \%$ of the selected events and have a positive $X$ in $(40 \pm 20) \%$ of the cases.

- CP-conserving $\mathrm{K}_{\mathrm{S}}^{0}$ decay amplitude The CP-conserving $\mathrm{K}_{\mathrm{S}}^{0} \rightarrow \pi^{+} \pi^{-} \pi^{0}$ decay amplitude does not cancel in the CP-violating asymmetry $\mathrm{A}_{+-0}(\tau)$ if there is any difference in the detector acceptance for the phasespace regions $X>0$ and $X<0$. The asymmetry of the acceptance was estimated by comparing the Dalitz plot distribution of real events with the one computed from the theoretical parametrization of the decay amplitudes $A_{\mathrm{L}}^{+-0}(X, Y)$ and $A_{\mathrm{S}}^{3 \pi(\mathrm{CP}=+1)}(X, Y)$ [7]. The systematic error on $\eta_{+-0}$ from this source is negligible.

- Decay-time dependence of normalization

The error introduced by the statistical uncertainties in the $\overline{\mathrm{K}}^{0} / \mathrm{K}^{0}$ normalization correction was taken into account. Although data were corrected for any time dependence of the normalization, a search for a residual effect due to different event topologies was carried out and found to lead to a small error.

\section{- Decay-time resolution}

The systematic errors due to finite decay-time resolution, bin size, and the lower limit of the decay-time interval used in fitting the asymmetries, were determined using simulated events.

\section{- Regeneration}

The effect of neutral kaon regeneration is expected to be negligible at short decay time, where the asymmetries are maximal. This uncertainty was found to be less than a few $10^{-5}$ when changing the regeneration amplitudes within the uncertainties of the values extrapolated from higher energy data $\left( \pm 13 \%\right.$ for the modulus and $\pm 9^{\circ}$ for the phase 
- $\Delta m, \Gamma_{\mathrm{S}}$ and $\Gamma_{\mathrm{L}}$

The experimental uncertainties on the values of $\Delta m, \Gamma_{\mathrm{S}}$ and $\Gamma_{\mathrm{L}}$ used in the fit have a negligible impact on the results, which are also insensitive to the input value of $\operatorname{Re}(\varepsilon)$, see Ref. [1].

- Acceptance

Owing to the separate integration over the phase space in the different parts of Eq. (1), the acceptance does not cancel in the expression for $\lambda$. Simulated data were used to determine the effect of the acceptance as a function of $X, Y$ and $\tau$.

\section{$5 \quad$ Final results and conclusions}

Our final result for $\eta_{+-0}$, shown in Fig. 4, is

$$
\begin{aligned}
& \operatorname{Re}\left(\eta_{+-0}\right)=\left(\begin{array}{l}
-2 \pm 7 \text { stat. } \\
\\
\operatorname{Im}\left(\eta_{+-0}\right)=\left(\begin{array}{l}
+4 \\
\text { syst. }
\end{array}\right) \times 10^{-3} \\
-2 \pm 9 \text { stat. } \\
-1 \\
-1
\end{array} \text { syst. }\right) \times 10^{-3} .
\end{aligned}
$$

Assuming no correlation between the systematic errors, we obtain $\left|\eta_{+-0}\right|<0.017$ at the $90 \%$ confidence level. Currently, this is the most precise determination of the real and imaginary parts of $\eta_{+-0}$.

Our final result for $\lambda$, shown in Fig. 5, is

$$
\begin{aligned}
& \operatorname{Re}(\lambda)=(+28 \pm 7 \text { stat. } \pm 3 \text { syst. }) \times 10^{-3} \\
& \operatorname{Im}(\lambda)=(-10 \pm 8 \text { stat. } \pm 2 \text { syst. }) \times 10^{-3} .
\end{aligned}
$$

We do not fix $\operatorname{Im}(\lambda)$ to zero, in order to take into account strong-interaction phase-shift differences between the $I=2$ and $I=1$ components of the decay amplitudes. The branching ratio for the CP-conserving $\mathrm{K}_{\mathrm{S}}^{0} \rightarrow \pi^{+} \pi^{-} \pi^{0}$ decay is estimated from $\operatorname{Re}(\lambda)$ and the $\mathrm{K}_{\mathrm{L}}^{0}$ decay parameters $[2,4]$. Neglecting second-order terms in the strong-interaction phase-shift differences, we obtained

$$
\mathrm{B}_{\mathrm{K}_{\mathrm{S}}^{0} \rightarrow \pi^{+} \pi^{-} \pi^{0}(\mathrm{CP}=+1)}=\left(2.5_{-1.0}^{+1.3} \text { stat. }{ }_{-0.6}^{+0.5} \text { syst. }\right) \times 10^{-7},
$$

where the systematic errors also include a contribution from the uncertainty on the $\mathrm{K}_{\mathrm{L}}^{0}$ decay parameters. Currently, these results are the most precise determination of the CP-conserving parameters for $\mathrm{K}_{\mathrm{S}}^{0}$ decays into $\pi^{+} \pi^{-} \pi^{0}$. These measurements are in good agreement with the values predicted both by phenomenological fits to all known kaon decay rates $[4,7]$ and by chiral perturbation theories [5-8].

\section{Acknowledgements}

We would like to thank the CERN LEAR staff as well as the technical and engineering staff of our institutes for their support and co-operation. This work was supported by the following institutions: the French CNRS/Institut National de Physique Nucléaire et de Physique des Particules, the French Commissariat à l'Energie Atomique, the Greek General Secretariat of Research and Technology, the Netherlands Foundation for Fundamental Research on Matter (FOM), the Portuguese JNICT, the Ministry of Science and Technology of the Republic of Slovenia, the Swedish Natural Science Research Council, the Swiss National Science Foundation, the UK Particle Physics and Astronomy Research Council (PPARC), and the US National Science Foundation. 


\section{References}

[1] R. Adler et al., Phys. Lett. B 370 (1996) 167

[2] R. Adler et al., Phys. Lett. B 374 (1996) 313

[3] R. Adler et al., CERN-PPE/96-189, 1996, to be published in Proc. Workshop on K Physics, Orsay, 1996

[4] T.J. Devlin and J.O. Dickey, Rev. Mod. Phys. 51 (1979) 237

[5] S. Fajfer and J.-M. Gérard, Z. Phys. C 42 (1989) 425

[6] H.-Y. Cheng, Phys. Lett. B 238 (1990) 399

[7] J. Kambor et al., Phys. Lett. B 261 (1991) 496

[8] G. D’Ambrosio et al., Phys. Rev. D 50 (1994) 5767; ibidem D 51 (1995) 3975

[9] R. Adler et al., Nucl. Instr. and Meth. A 379 (1996) 76

[10] R. Adler at al., Phys. Lett. B 369 (1996) 367; updated with CPLEAR full statistics, to be published.

[11] R.H. Barnett et al., Phys. Rev. D 54 (1996) 1

[12] R. Adler et al., Phys. Lett. B 363 (1995) 243

[13] Y. Zou et al., Phys. Lett. B 329 (1994) 519

[14] Y. Zou et al., Phys. Lett. B 369 (1996) 362 


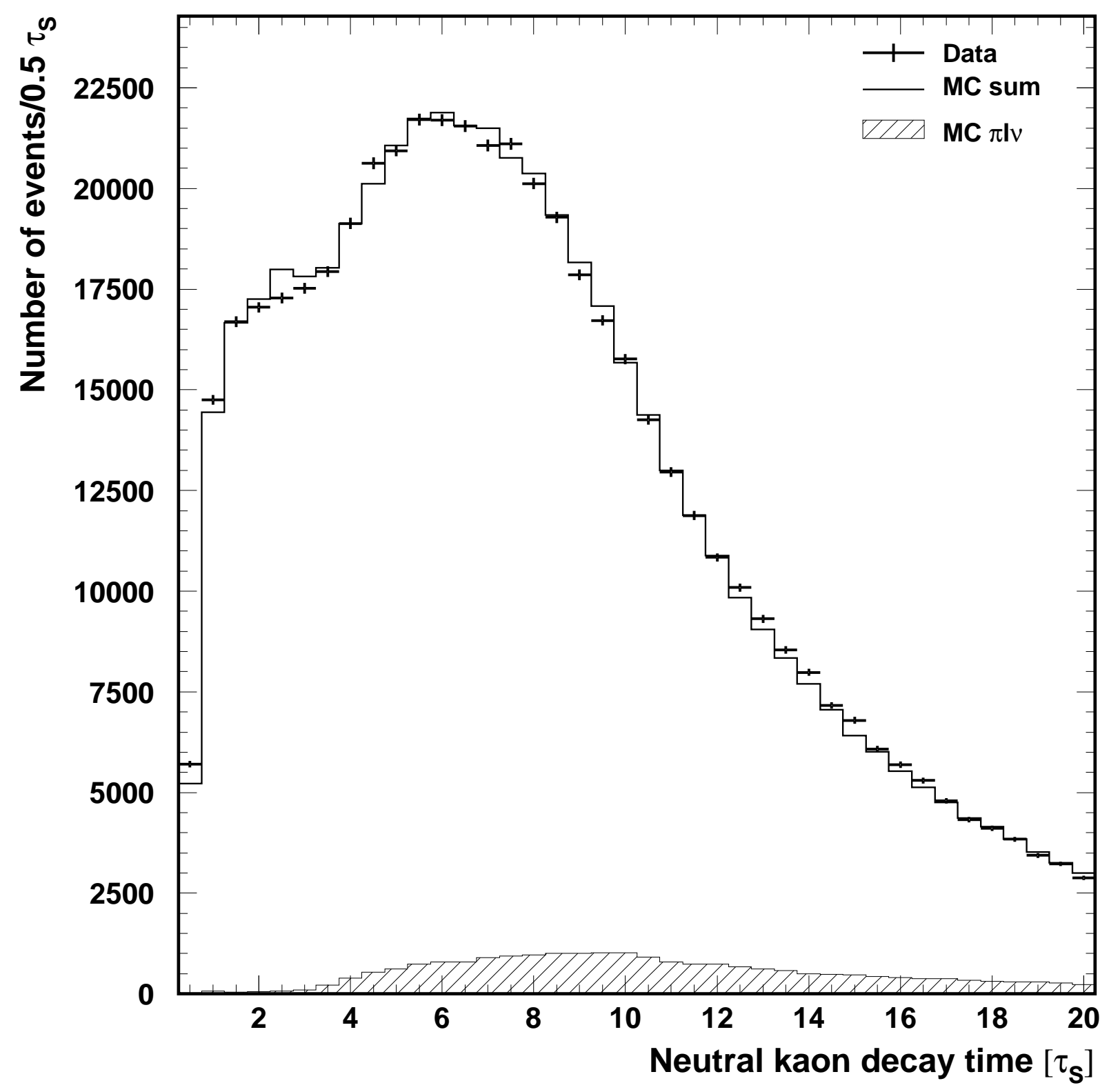

Figure 1: The decay-time distribution of initial $\mathrm{K}^{0}$ and $\overline{\mathrm{K}}^{0}$ decaying into $\pi^{+} \pi^{-} \pi^{0}$ for real (+) and simulated (-) data. The simulated distribution (MC sum) results from $\pi^{+} \pi^{-} \pi^{0}$ and semileptonic decays of neutral kaons and is normalized to the real data set above $6 \tau_{\mathrm{S}}$. The background contribution from semileptonic events is given by the shaded area. 


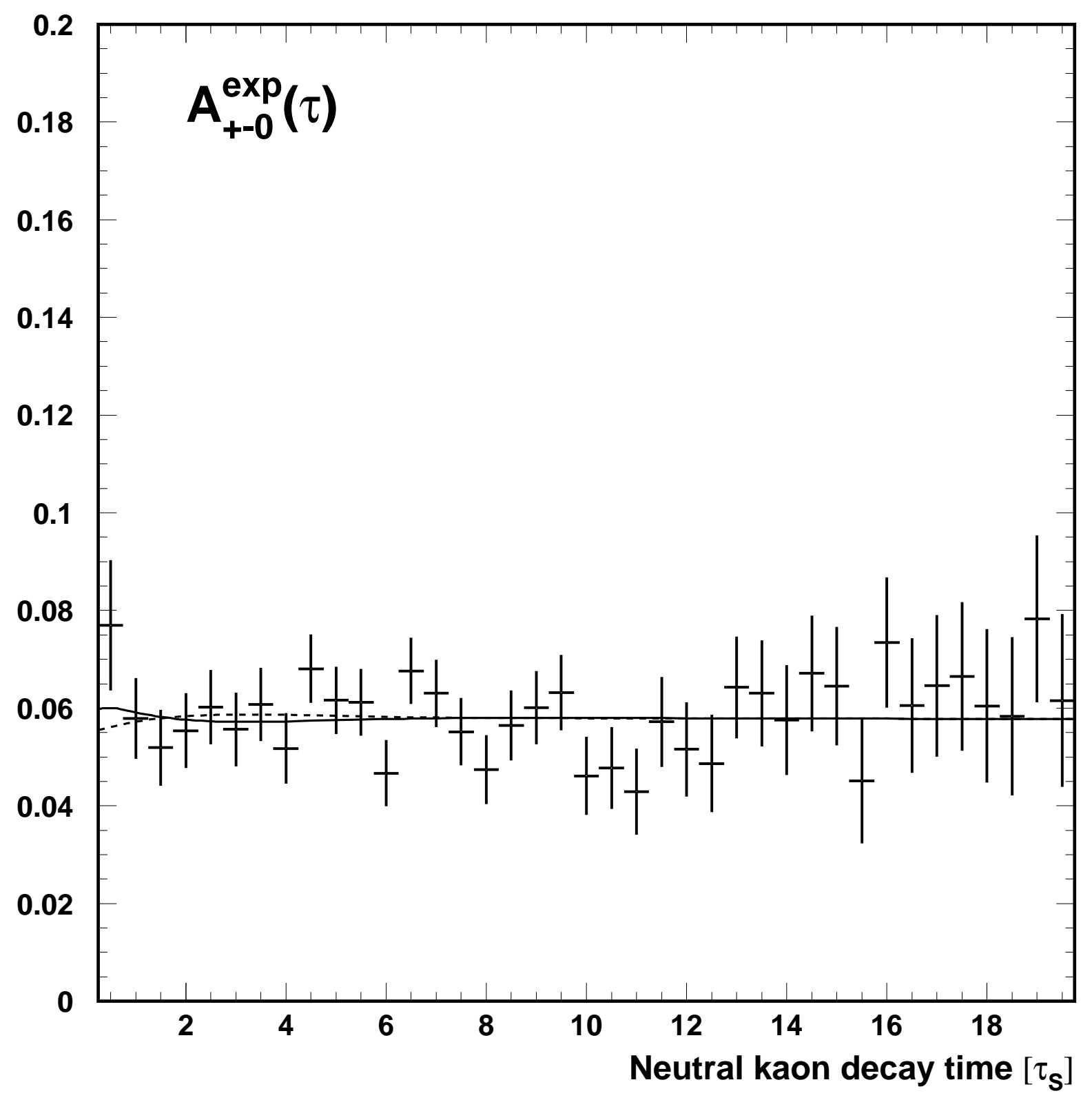

Figure 2: The measured CP-violating decay-rate asymmetry between 0.25 and $19.75 \tau_{\mathrm{S}}$. The solid line is obtained by fitting Eq. (4) to the data. The broken line shows the asymmetry expected when assuming $\eta_{+-0}=\eta_{+-}$. 

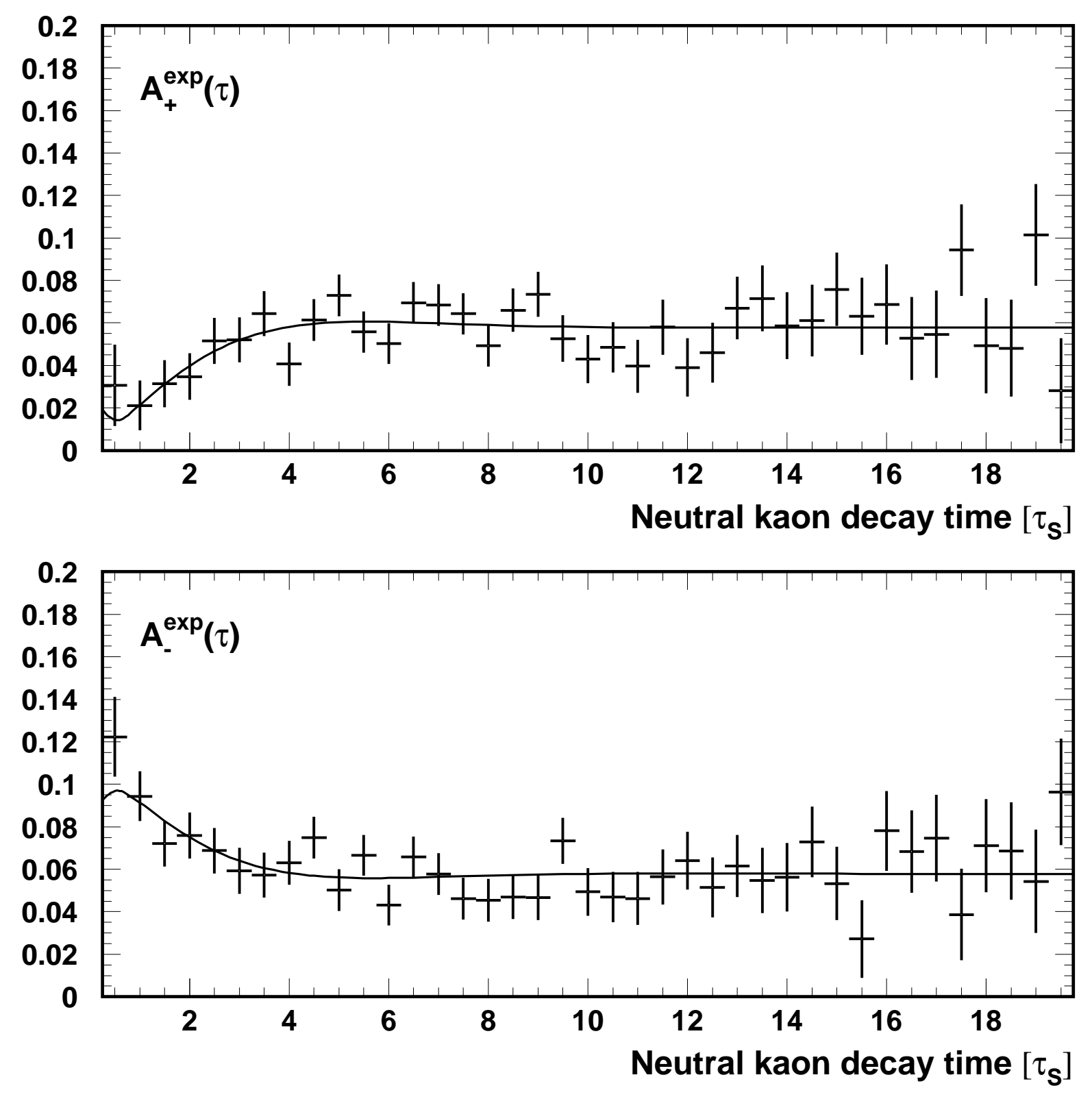

Figure 3: The measured decay rate asymmetries for $X>0$ and $X<0$ between 0.25 and $19.75 \tau_{\mathrm{S}}$. The solid curves are the result of the simultaneous fit of Eq. (6) to the data, assuming common $\lambda$ and $\xi$. In this determination, we fixed $\eta_{+-0}=\eta_{+-}$. 


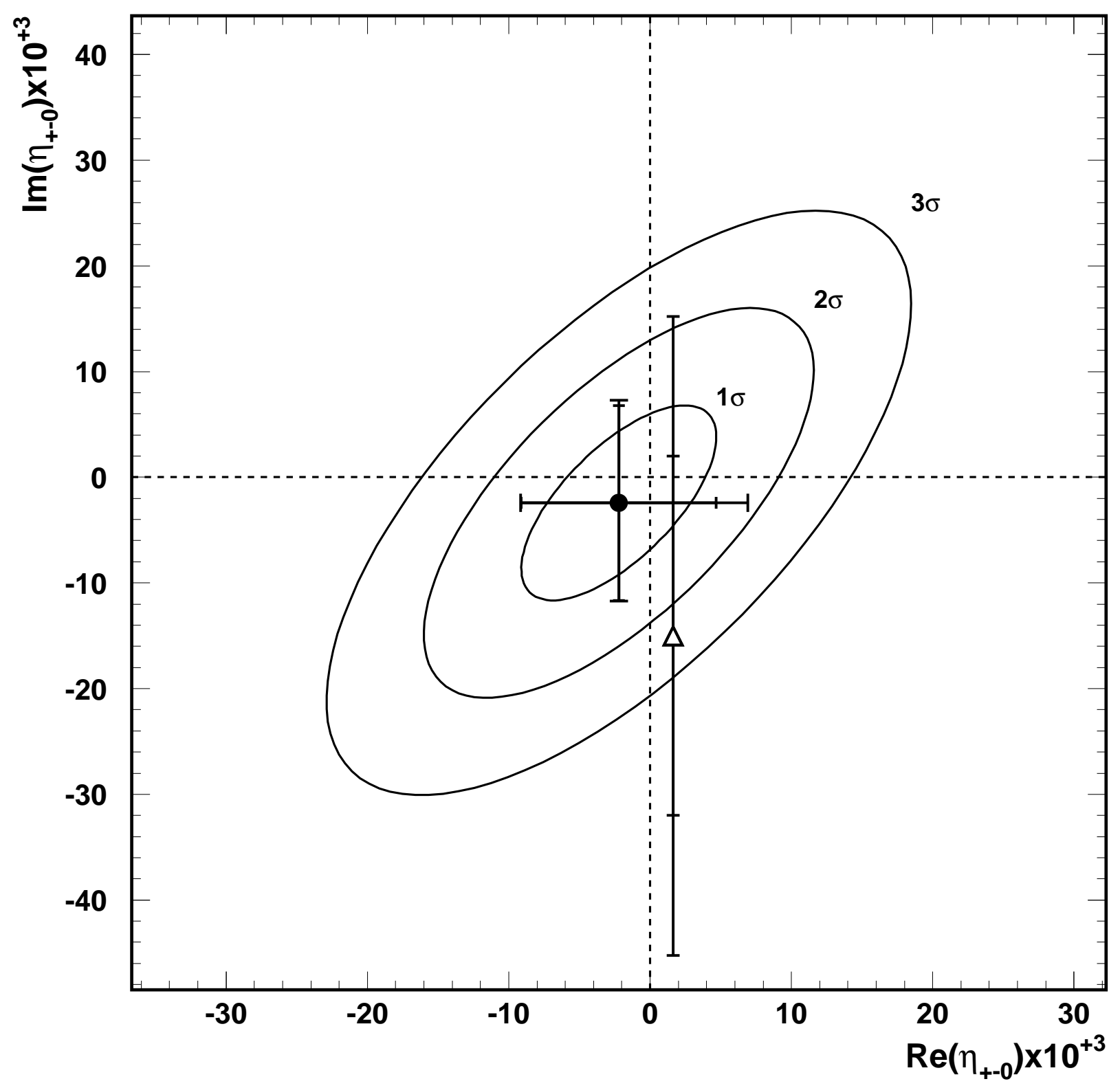

Figure 4: The real and the imaginary parts of $\eta_{+-0}:$ the central value $(\bullet)$ with statistical and total uncertainties, and the statistical contour plot; this paper. For comparison, the result of Zou et al. [13] on $\operatorname{Im}\left(\eta_{+-0}\right)$ obtained by fixing $\operatorname{Re}\left(\eta_{+-0}\right)=\operatorname{Re}(\varepsilon)$ is also shown $(\triangle)$. 


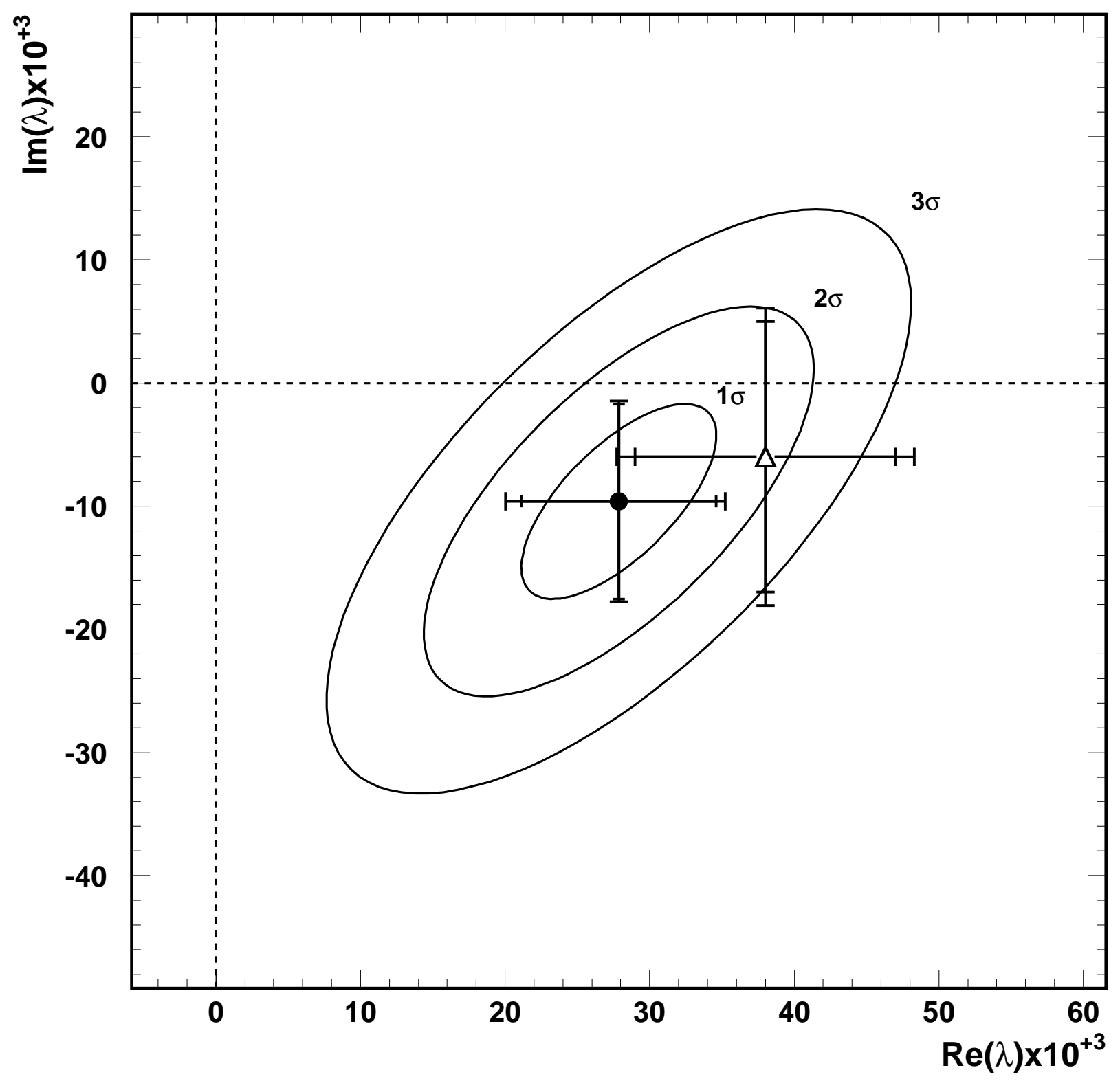

Figure 5: The real and the imaginary parts of $\lambda$ : the central value $(\bullet)$ with statistical and total uncertainties, and the statistical contour plot; this paper. For comparison, the result of Zou et al. $[14]$ is also shown $(\triangle)$. 